\title{
DNA: Building Blocks of Life Under UV Irradiation
}

\author{
Thomas Gustavsson, Roberto Improta* and Dimitra Markovitsi \\ Laboratoire Francis Perrin, CEA/DSM/IRAMIS/SPAM - CNRS URA 2453, CEA/Saclay, \\ 91191 Gif-sur-Yvette, France \\ * Istituto Biostrutture e Bioimmagini - CNR, Via Mezzocannone 16, 80134 Napoli, Italy
}

\begin{abstract}
During the last ten years, intense experimental and theoretical work has proven the existence of ultrafast non-radiative decay routes for UV-excited monomeric nucleic acid bases explaining their high photostability. This mechanism has been explained by the occurrence of easily accessible conical intersections connecting the first excited $\pi \pi^{*}$ state with the ground state. However, recent studies of substituent and solvent effects indicate that the situation is more complicated than what was initially thought, notably by the presence of dark excited states. Moreover, the actual shape of the excited state potential energy surface may induce non-exponential dynamics. Further efforts are needed in order to clarify how various environmental factors affect the structural and dynamical aspects of the nucleic acid base excited states.
\end{abstract}


TOC Figure

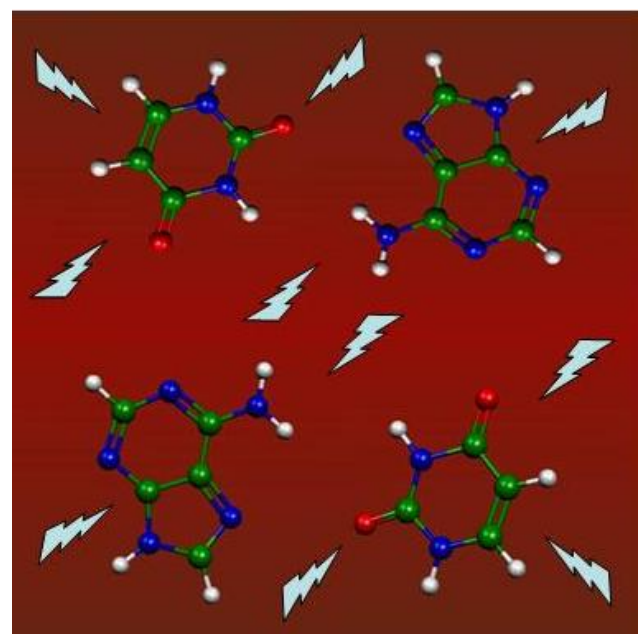

Keywords: DNA, nucleic acid bases, femtosecond, fluorescence, internal conversion, conical intersection 
To this date, the exact circumstances for the beginning of life on Earth remain obscure. However, much of the prebiotic chemistry probably occurred under intense UV irradiation. Among the various molecules that emerged at that time, the purines (adenine and guanine) and pyrimidines (uracil, thymine, and cytosine) that became the building blocks of RNA and DNA, possess a remarkable photostability. Indeed, in spite of the fact that these molecules absorb strongly in the UV, the excess energy is rapidly dissipated to the surroundings in form of heat, as shown by the very low fluorescence quantum yield suggesting excited state lifetimes shorter than 1 picosecond $\left(10^{-12} \mathrm{~s}\right)$. This natural protection of the nucleic acid bases against UV light has come to be considered the key for their success.

Ultrafast and effective non-radiative decay routes exist for the electronically excited states of all nucleic acid bases. Is this the reason for them being selected as fundamental building blocks of life?

Obtaining a detailed picture of the static and the dynamical behavior of the electronically excited states of nucleic acid bases, nucleosides and nucleotides (NABs) and assessing the molecular mechanisms underlying their very efficient nonradiative deactivation is thus important. In addition to the intrinsic interest of these molecules, unveiling the photophysical and photochemical behaviour of the DNA/RNA monomeric constituents is mandatory for a full comprehension of the phenomena triggered in single- and doublestranded nucleic acids by the absorption of UV-light. These processes are biologically relevant since the initially populated singlet excited electronic states may constitute the first step of a cascade of events leading to photochemical reactions responsible for carcinogenic mutations. 
On these grounds, it is not surprising that a rapidly growing amount of experimental and computational studies has been devoted to the electronically excited states of the NABs during the last decade. As a consequence, they have become molecular "guinea pigs" for assessing the basic physical-chemical effects ruling the non-radiative decay of organic molecules in general. A detailed review of the situation up to 2004 can be found in ref. 1 .

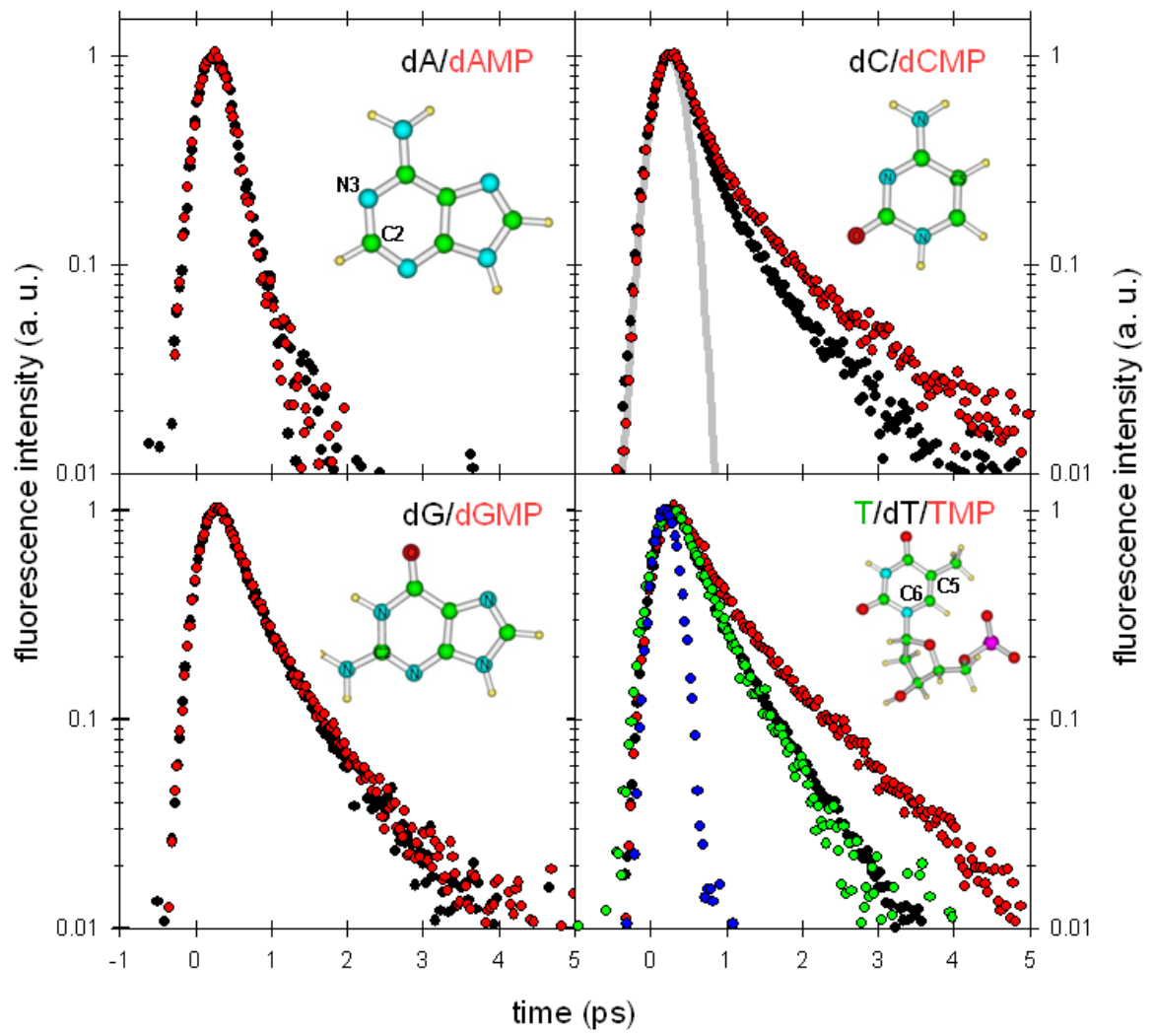

Figure 1. Fluorescence decays for the four nucleosides (black) and four nucleotides (red). Also shown are the fluorescence decays of thymine (green) and uracil (blue) as well as the apparatus function (gray line).

The structures of adenine, cytosine, guanine and thymidine monophosphate are presented in the corresponding panels. The average lifetimes are : $0.13 / 0.13 \mathrm{ps}(\mathrm{dA} / \mathrm{dAMP}), 0.30 / 0.45 \mathrm{ps}(\mathrm{dC} / \mathrm{dCMP})$, $0.33 / 0.34 \mathrm{ps}$ (dG/dGMP), $0.30 / 0.32 / 0.50 \mathrm{ps}$ (T/dT/TMP) and <0.1 ps (U).

On the experimental side a major issue was how to monitor the time-evolution of the first singlet excited state population (in the following denoted $S_{\pi \pi^{*}}$ ) with sufficiently high time-resolution. A decisive step forward was the successful use of UV femtosecond laser spectroscopic techniques in the characterisation of the NABs. In the gas phase, femtosecond two-photon ionisation/REMPI experiments provided clear evidence for ultrafast $(<1 \mathrm{ps})$ 
excited state deactivation processes although slower components have also been observed. ${ }^{2}$ In solution, most results have been obtained by two pump-probe techniques; fluorescence upconversion (FU) and transient absorption (TA). The former probes the $S_{\pi \pi^{*}}$ spontaneous emission and the latter the $S_{\pi \pi^{*}} \rightarrow S_{n}$ induced absorption, where $S_{n}$ is a higher excited singlet state. At the beginning of the millennium these techniques had proven invaluable tools in numerous studies of other molecular systems, but they were poorly adapted to NAB spectroscopy falling into the UV spectral region. The real breakthroughs were the discovery of a $S_{\pi \pi^{*}} \rightarrow S_{n}$ TA band in the visible spectral region and the adaptation of FU in the UV, allowing the monitoring of the NAB $S_{\pi \pi^{*}}$ population. Both techniques have been successfully applied to various NABs allowing the first determinations of the $S_{\pi \pi^{*}}$ excited state lifetimes. ${ }^{3-5}$ The main outcome of these and subsequent experimental studies was that the excited state decays were indeed found to be much faster than $1 \mathrm{ps}$, for all the NABs. ${ }^{6-11}$ To illustrate this, fluorescence decays for the eight nucleosides and nucleotides are shown in Figure 1.

Additional experimental information has also been obtained by picosecond UV/infrared transient absorption spectroscopy. ${ }^{12}$ Probing in the $1500-2000 \mathrm{~cm}^{-1}$ range after UV excitation brought information not only about the "hot ground state" but also a longerlived state in the case of 2'-deoxycytidine 5'-monophosphate (dCMP). Furthermore, resonance Raman experiments have shown shown that uracil-like molecules keep a planar geometry during the first femtoseconds after the excitation of $\mathrm{S}_{\pi, .}{ }^{13}$ Triggered by the increasing amount of experimental findings, quantum chemistry calculations at various levels were dedicated to the study of the excited state behavior of the NABs and have provided a very rich source for understanding the mechanisms at the origin for their exceedingly short lifetimes ${ }^{7,10,14-22}$

The picture emerging from these experimental and theoretical studies is that the ultrafast decay of the first singlet excited state $S_{\pi \pi^{*}}$ is due to highly efficient conical 
intersections $(\mathrm{CI})$ between this state and the ground state $\left(\mathrm{S}_{0}\right)$. Very loosely speaking, for the pyrimidines, the internal conversion through the $\mathrm{CI}$ involves a pyramidalization of $\mathrm{C}_{5}$ and/or a torsion of the $\mathrm{C}_{5} \mathrm{C}_{6}$ bond accompanied by an out-of-plane motion of the 5-substituent (see Figure 1 for atom labelling). For the purines, the reaction path involves a twisting around the $\mathrm{C}_{2} \mathrm{~N}_{3}$ bond provoking an out-of-plane bending of the 2-substituent. Such a general description is bound to be oversimplified, and a very active debate (a review of which is outside the scope of this perspective) exists regarding the details of the decay mechanism for each individual base. In particular, the decay mechanism of cytosine is far from being completely assessed. On the other hand, theoretical studies agree in predicting that almost barrierless paths connect the Franck-Condon region of the $S_{\pi \pi^{*}}$ state with the CI with $S_{0}$ for all the NAB's, accounting for their ultrafast decay. ${ }^{23}$ This scenario, originally proposed on the ground of gas phase calculations, is to a large extent also valid in solution. As an example, Figure 2 illustrates the UV-induced structural changes occurring in uracil in water and leading to the CI.

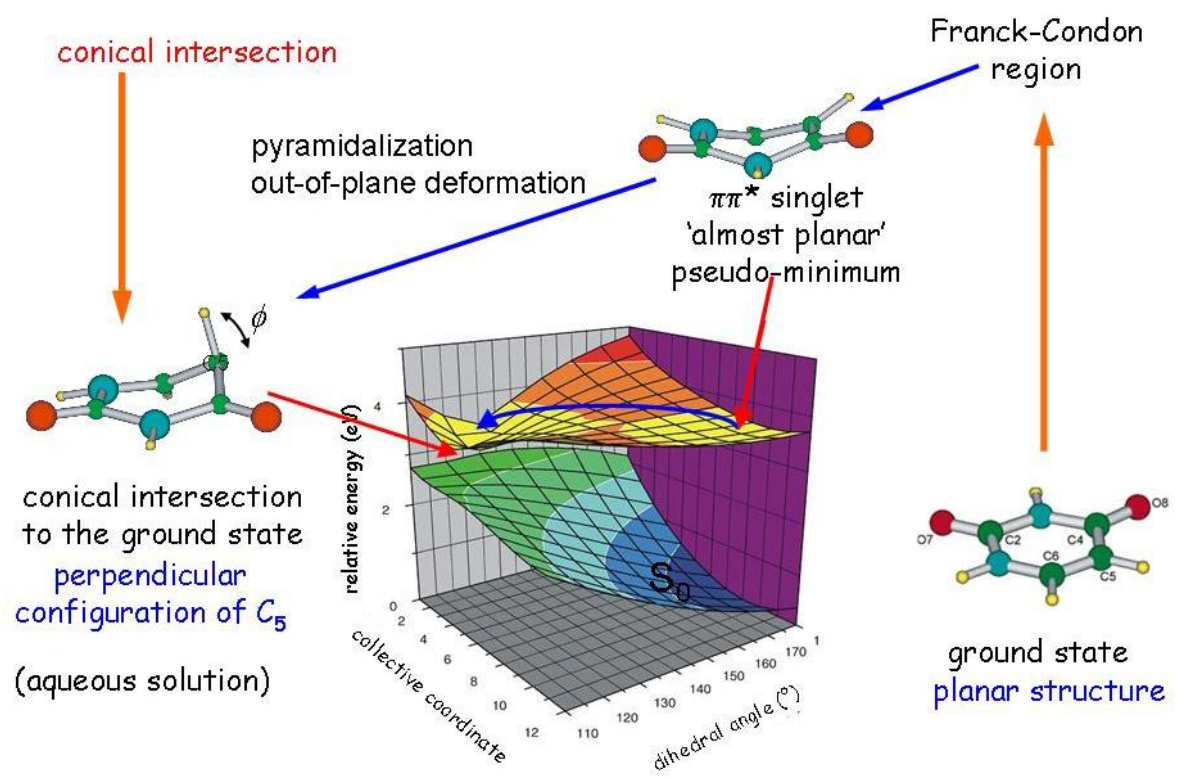

Figure 2. The picture emerging today for the uracil $S_{\pi \pi^{*}}$ excited state dynamics in aqueous solution. The initial evolution from a flat structure in the Franck-Condon towards a flat pseudo-minimum region is followed by a very rapid ( $<100 \mathrm{fs}$ ) out of plane motion driving the system to a conical intersection point with the ground state where a very efficient internal conversion takes place. Similar scenarios apply to the other NABs. (Potential energy surfaces taken from ref. 10) 
The very efficient internal conversion is explained by the existence of easily accessible conical intersections connecting the first excited $\pi \pi^{*}$ state with the ground state.

According to this simple and easy-to-grasp representation, the excited state decay of monomeric NABs is ruled by intrinsic effects involving the properties of the lowest energy bright state $\mathrm{S}_{\pi *}$ only. Although such a description can be used as a first approach, the most recent experimental and computational studies revealed a much more intricate picture. Below we highlight some factors that contribute to this increased complexity.

First, for all NABs, a spectroscopically dark excited state with $n \pi^{*}$ character $\left(S_{n} \pi^{*}\right)$ is very close in energy to the bright $S_{\pi \pi^{*}}$ state in the Franck-Condon region. Consequently, besides the direct $S_{\pi \pi^{*}} \rightarrow S_{0}$ decay (radiative or non-radiative), $S_{\pi \pi^{*}} \rightarrow S_{n \pi^{*}}$ and $S_{n \pi^{*}} \rightarrow S_{0}$ population transfers also influence the excited state deactivation. Indeed, if for uracil and thymine the involvement of a dark $S_{n \pi^{*}}$ state in the gas phase dynamics is still a matter of debate, in solution, experiments and computations agree in predicting a fast and effective $\mathrm{S}_{\pi \pi^{*} \rightarrow} \mathrm{S}_{\mathrm{n} \pi^{*}}$ population transfer. The dark $\mathrm{S}_{\mathrm{n} \pi^{*}}$ state is characterized by a slower decay component ( $25 \mathrm{ps})$, as measured by TA. ${ }^{24}$ The relative energies of the bright $\mathrm{S}_{\pi \pi^{*}}$ and the dark $S_{n \pi^{*}}$ excited states are strongly tuned by the environment, giving rise to significant solvent effects in the excited state dynamics of NABs. For example, the excited state lifetime of thymine decreases from 0.39 to 0.24 picoseconds when going from water to acetonitrile, while a fourfold decrease is observed for 5-fluorouracil (from 1.39 to $0.36 \mathrm{ps}$ ). ${ }^{10,15}$ Interestingly, the lifetimes of the $S_{\pi \pi^{*}}$ excited state of uracils in alcohols were found to be intermediate between those determined for water and acetonitrile solutions. These findings have been fully rationalized by quantum mechanical calculations including the solvent, which show that the stability of the $\mathrm{S}_{\mathrm{n} \pi^{*}}$ decreases significantly in hydrogen bonding media. As a result, the energy gap between $S_{n \pi^{*}}$ and $S_{\pi \pi^{*}}$ is modified affecting the deactivation process. ${ }^{25}$ 
This is illustrated in Figure 3 where fluorescence decays and potential energy surfaces of 5fluorouracil in protic and aprotic polar solvents are shown.
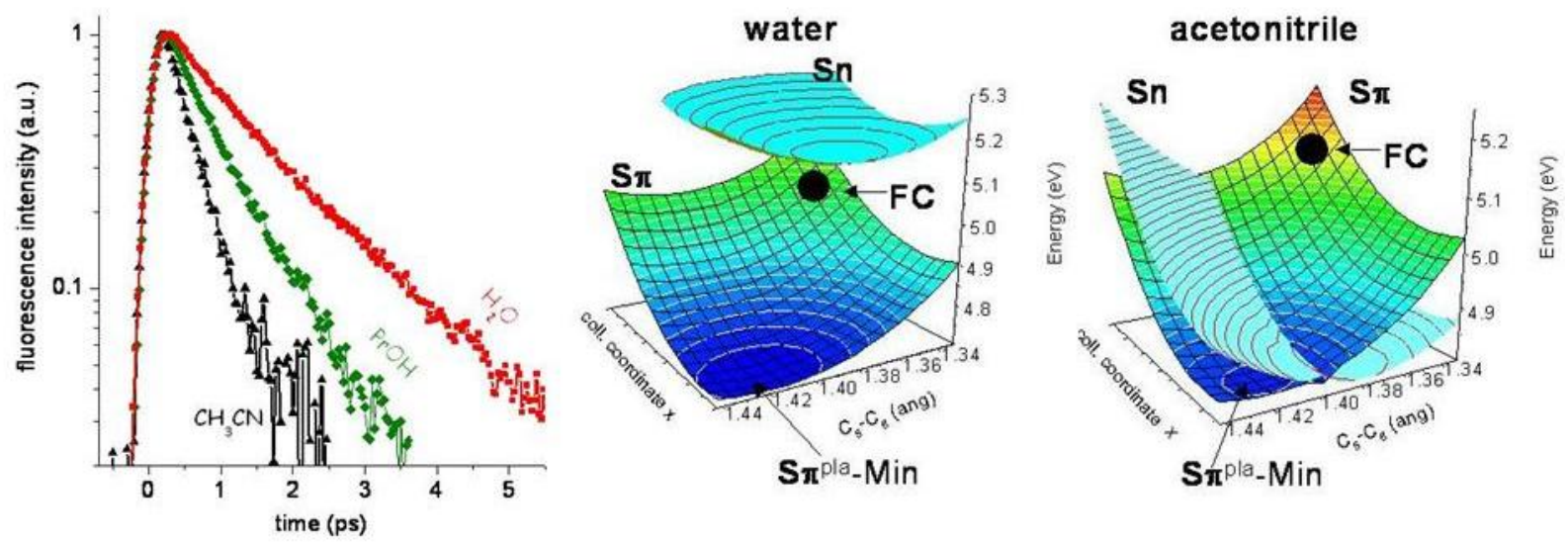

Figure 3. Solvent dependence of the 5 -fluorouracil excited state dynamics: fluorescence decays in water, propanol and acetonitrile (left), potential energy surfaces for the $S_{\pi \pi}{ }^{*}$ and $S_{n \pi}{ }^{*}$ states in water (middle) and in acetonitrile (right). (Potential energy surfaces taken from ref. 25)

Another source of complexity is the fact that, on the femtosecond timescale, the initially excited $S_{\pi \pi^{*}}$ state population is strongly out of equilibrium corresponding to a rapidly evolving wavepacket. For this reason, the excited state decays of NABs cannot be fittted by mono-exponential functions. We underline that such non-exponential decays are not necessarily due to the involvement of several electronically excited states. Two detailed studies of guanosine $5^{\prime}$-monophosphate $(\mathrm{GMP})^{7}$ and 2'-deoxyguanosine 5'-monophosphate (dGMP) ${ }^{26}$ show clearly that the correspondence "one time constant - one electronic state" does not hold. Contrary to what had been observed for the other NABs, the dGMP fluorescence decays were found to depend strongly on the emission wavelength, getting longer when going towards the red. This was explained by theoretical calculations predicting an excited state surface being practically flat along the reaction coordinate towards the CI. The wavepacket motion undergoes a "spreading out", producing strongly wavelength dependent dynamics from the same excited state potential energy surface, as illustrated in Figure 4. Actually, there are several hints that also for other NABs the excited state dynamics 
is modulated by the interplay between planar and non planar region of the $S_{\pi \pi^{*}}$ potential energy surface. $^{25,27}$

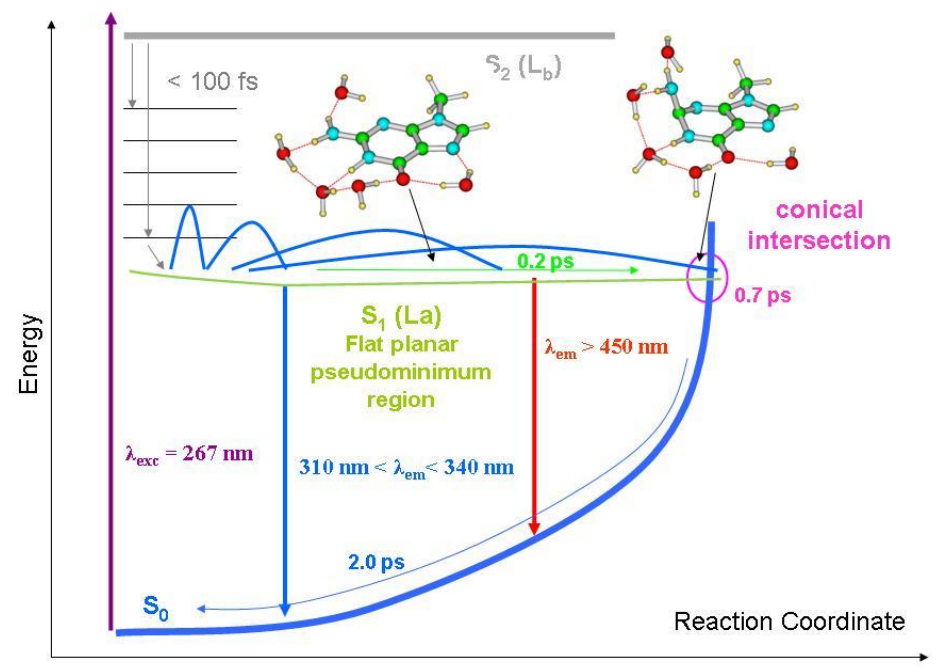

Figure 4. Schematic picture illustrating the wavepacket dynamics on a "flat" excited state potential energy surface. The rapid evolution from a localized well-defined function in the Franck-Condon region towards a delocalized, diffuse distribution at longer time.

Fast does not mean simple: dark excited states, interactions with the solvent, ring substituents, topology of the potential energy surfaces.....the excited state decays of NABs can be very complex.

A very useful method to shed light on such complex decay mechanisms is a systematic exploitation of substituent effect. ${ }^{10}$ Replacing one particular hydrogen or methyl group on the NAB skeleton by another substituent group may have dramatic effects on the excited state dynamics. The most representative example is the difference in excited state decay rate in water between the RNA base uracil $\left(>10^{13} \mathrm{~s}^{-1}\right)$ and the DNA base thymine $\left(5 \cdot 10^{13} \mathrm{~s}^{-1}\right)$, where the hydrogen in the 5-position is replaced by a methyl group. Moreover, the excited state lifetime of the minor natural base 5-methylcytosine, is about ten times longer than that of cytosine. ${ }^{28}$ These two examples provided an unambiguous proof of the involvement of the $\mathrm{C}_{5}$ moiety in the non-radiative deactivation mechanism in pyrimidines. However, other more 
subtle substituent effects remain to elucidate. One striking example is the effect of the deoxyribose and of the phosphate group on the NBAs excited state dynamics, which is apparent for pyrimidines while it is negligible for the purines (Figure 1). ${ }^{29}$ This is surprising taking into account the fact that the steady-state fluorescence spectra of nucleosides and nucleotides are identical. Moreover, quantum mechanical computations suggest that the main features of the lowest energy excited states of the nucleosides and nucleotides are similar to those of the bases in the FC region. ${ }^{22}$

There may be different explanations for the way the phosphate group affects the pyrimidine photophysical behavior. One can mention the formation of additional hydrogen bonds which could be favored by the folding of the flexible group or the presence of a negative charge in the vicinity of the chromophore. Experiments also suggest that the ionic strength of the solution affects the pyrimidine excited state dynamics. ${ }^{29,30}$ Elucidating the role of local charges on the excited state behaviour is crucial for obtaining a correct description of the photoprocesses within the double helix which is a polyelectrolyte. In order to bridge the gap between our knowledge of the isolated NABs and that of DNA double strands, ${ }^{31,32}$ we should discriminate between the collective features and those only due to the environmental effects on the behaviour of monomeric NAB. To this aim, it would be helpful to study the NABs in conditions not too different from a DNA-like environment.

There are several other open questions whose elucidation is not only important for getting the full picture of the NABs excited state decays but is also crucial for the understanding of DNA behaviour. They concern not only the bright $S_{\pi \pi^{*}}$ states but also the dark excited states. For example, a 30 ps lifetime detected in the infrared absorption spectrum of the poly $(\mathrm{dGdC}) \cdot$ poly $(\mathrm{dGdC})$ duplex was assigned to the $\mathrm{n} \pi^{*}$ state localized on cytosine. ${ }^{33}$ The steady-state fluorescence spectrum of the same duplex is dominated by emission from “dark" states with nanosecond lifetime. ${ }^{34}$ Other dark states, $\pi \sigma^{*}$ states, have also been 
proposed to influence the excited state dynamics in the gas phase. ${ }^{35}$ Their dependence on the environmental factors is also an important issue. It is also possible that "dark" states may borrow intensity from close-lying bright states and fluoresce weakly in solution. In this respect, experiments using time-correlated single photon counting, which is much more sensitive than FU, would be precious.

Another aspect which merits closer attention is the characterization of the triplet states. Intersystem crossing quantum yields in purines and pyrimidines were determined experimentally decades ago, ${ }^{36,37}$ but it would be instructive to revisit this topic in the light of the accumulated knowledge of the singlet excited state described above. Recently, results from a time-resolved infrared study of the lowest triplet state of thymine and thymidine were interpreted as the triplet state being fully formed within the first 10 ps after excitation. ${ }^{38}$

On the theoretical side, a real breakthrough would be the development and the applications of suitable quantum dynamical methods which have just started to be successfully applied to the study of NAB excited states. ${ }^{20,27}$ Such methods would allow a direct quantitative comparison with results from time-resolved spectroscopy such as time constants and branching ratios.

In conclusion, giant steps have been made in the last few years towards the understanding of the NAB excited state deactivation mechanism, and the general features seem to be well understood. On the other hand, as we have just highlighted, many fundamental questions remain to be answered. We are convinced of the importance of continued joint experimental and theoretical efforts and, in particular, systematic studies of environmental and substitutional effects. Although femtosecond spectroscopic techniques remain the key tool in this field, it is also important to explore the above-mentioned effects on longer times. Theoretical models should provide a valid description for the ensemble of experimental data. 


\section{References}

(1) Crespo-Hernández, C. E.; Cohen, B.; Hare, P. M.; Kohler, B. Ultrafast ExcitedState Dynamics in Nucleic Acids, Chem. Rev. 2004, 104, 1977 -2020.

(2) Canuel, C.; Mons, M.; Piuzzi, F.; Tardivel, B.; Dimicoli, I.; Elhanine, M. Excited States Dynamics of DNA and RNA Bases: Characterization of a Stepwise Deactivation Pathway in the Gas Phase, J. Chem. Phys. 2005, 122, $0743161-0743166$.

(3) Pecourt, J.-M. L.; Peon, J.; Kohler, B. Ultrafast Internal Conversion of Electronically Excited RNA and DNA Nucleosides in Water, J. Am. Chem. Soc. 2000, 122, 9348-9349.

(4) Peon, J.; Zewail, A. H. DNA/RNA Nucleotides and Nucleosides: Direct Measurement of Excited-state Lifetimes by Femtosecond Fluorescence Up-conversion, Chem. Phys. Lett. 2001, 348, 255-262.

(5) Gustavsson, T.; Sharonov, A.; Markovitsi, D. Thymine, Thymidine and Thymidine 5'-monophosphate Studied by Femtosecond Fluorescence Upconversion spectroscopy, Chem. Phys. Lett. 2002, 351, 195-200.

(6) Pancur, T.; Schwalb, N. K.; Renth, F.; Temps, F. Femtosecond Fluorescence Up-conversion Spectroscopy of Adenine and Adenosine: Experimental Evidence for the $\pi \sigma^{*}$ State? Chem. Phys. 2005, 313, 199-212.

(7) Karunakaran, V.; Kleinermanns, K.; Improta, R.; Kovalenko, S. A. Photoinduced Dynamics of Guanosine Monophosphate in Water from Broad-Band Transient Absorption Spectroscopy and Quantum-Chemical Calculations, J. Am. Chem. Soc. 2009, 131, 5839-5850.

(8) Sharonov, A.; Gustavsson, T.; Carré, V.; Renault, E.; Markovitsi, D. Cytosine Excited State Dynamics Studied by Femtosecond Spectroscopy, Chem. Phys. Lett. 2003, $380,173-180$.

(9) Blancafort, L.; Cohen, B.; Hare, P. M.; Kohler, B.; Robb, M. A. Singlet ExcitedState Dynamics of 5-Fluorocytosine and Cytosine: An Experimental and Computational Study, J. Phys. Chem. A 2005, 109, 4431-4436.

(10) Gustavsson, T.; Banyasz, A.; Lazzarotto, E.; Markovitsi, D.; Scalmani, G.; Frisch, M. J.; Barone, V.; Improta, R. Singlet Excited-state Behavior of Uracil and Thymine in Aqueous Solution: A Combined Experimental and Computational Study of 11 Uracil Derivatives, J. Am. Chem. Soc. 2006, 128, 607-619.

(11) Kwok, W.-M.; Ma, C.; Phillips, D. L. A Doorway State Leads to Photostability or Triplet Photodamage in Thymine DNA, J. Am. Chem. Soc. 2008, 130, 5131-5139.

(12) Towrie, M.; Doorley, G. W.; George, M. W.; Parker, A. W.; Quinn, S. J.; Kelly, J. M. ps-TRIR Covers All the Bases - Recent Advances in the Use of Transient IR for the Detection Of Short-Lived Species in Nucleic Acids, Analyst 2009, 134, 1265-1273.

(13) Billinghurst, B. E.; Yeung, R.; Loppnow, G. R. Excited-State Structural Dynamics of 5-Fluorouracil, J. Phys. Chem. A 2006, 110, 6185-6191.

(14) Matsika, S. Radiationless Decay of Excited States of Uracil through Conical Intersections, J. Phys. Chem. A 2004, 108, 7584-7590.

(15) Gustavsson, T.; Sarkar, N.; Lazzarotto, E.; Markovitsi, D.; Improta, R. Singlet Excited State Dynamics of Uracil and Thymine Derivatives. A Femtosecond Fluorescence Upconversion Study in Acetonitrile, Chem. Phys. Lett. 2006, 429, 551-557. 
(16) Merchán, M.; Gonzalez-Luque, R.; Climent, T.; Serrano-Andres, L.; Rodriguez, E.; Reguero, M.; Pelaez, D. Unified Model for the Ultrafast Decay of Pyrimidine Nucleobases, J. Phys. Chem. B 2006, 110, 26471-26476.

(17) Mercier, Y.; Santoro, F.; Reguero, M.; Improta, R. The Decay From The Dark $\mathrm{n} \pi *$ Excited State in Uracil: An Integrated CASPT2/CASSCF and PCM/TD-DFT Study in the Gas Phase and in Water, J. Phys. Chem. B 2008, 112, 10769-10772.

(18) Santoro, F.; Improta, R.; Barone, V. Three-dimensional Diabatic Models for the $\pi \pi^{*} \rightarrow \mathrm{n} \pi^{*}$ Excited-state Decay of Uracil Derivatives in Solution, Theoretical Chemistry Accounts 2009, 123, 273-286.

(19) Blancafort, L. Energetics of Cytosine Singlet Excited-State Decay Paths; A Difficult Case for CASSCF and CASPT2, Photochem. Photobiol. 2007, 83, 603-610.

(20) Hudock, H. R.; Martinez, T. J. Excited-State Dynamics of Cytosine Reveal Multiple Intrinsic Subpicosecond Pathways, ChemPhysChem 2008, 9, 2486-2490.

(21) Conti, I.; Garavelli, M.; Orlandi, G. Deciphering Low Energy Deactivation Channels in Adenine, J. Am. Chem. Soc. 2009, 131, 16108-16118.

(22) Improta, R.; Barone, V. The Excited States of Adenine and Thymine Nucleoside and Nucleotide in Aqueous Solution: A Comparative Study by Time-dependent DFT Calculations, Theoretical Chemistry Accounts 2008, 120, 491-497.

(23) Serrano-Andrés, L.; Merchán, M. Are the Five Natural DNA/RNA Base Monomers a Good Choice from Natural Selection? A Photochemical Perspective, J. Photochem. Photobiol. C: Photohemistry Reviews 2009, 10, 21-32.

(24) Hare, P. M.; Crespo-Hernández, C. E.; Kohler, B. Solvent-Dependent Photophysics of 1-Cyclohexyluracil: Ultrafast Branching in the Initial Bright State Leads Nonradiatively to the Electronic Ground State and a Long-Lived ${ }^{1} n \pi{ }^{*}$ State, J. Phys. Chem. B 2006, 110, 18641-18650.

(25) Santoro, F.; Barone, V.; Gustavsson, T.; Improta, R. Solvent Effect on the Singlet Excited State Lifetimes of Nucleic Acid Bases: A Computational Study of 5Fluorouracil and Uracil in Acetonitrile and Water, J. Am. Chem. Soc. 2006, 128, 16312-16322.

(26) Miannay, F.-A.; Gustavsson, T.; Banyasz, A.; Markovitsi, D. Excited State Dynamics of dGMP Measured by Steady-state and Femtosecond Fluorescence Spectroscopy, J. Phys. Chem. A 2010, 114, 3256-3263.

(27) Improta, R.; Barone, V.; Lami, A.; Santoro, F. Quantum Dynamics of the Ultrafast $\pi \pi^{*} / \mathrm{n} \pi *$ Population Transfer in Uracil and 5 -Fluoro-Uracil in Water and Acetonitrile, J. Phys. Chem. B 2009, 113, 14491-14503.

(28) Sharonov, A.; Gustavsson, T.; Marguet, S.; Markovitsi, D. Photophysical Properties of 5-Methylcytosine, Photochem. Photobiol. Sci. 2003, 2, 1-5.

(29) Onidas, D.; Markovitsi, D.; Marguet, S.; Sharonov, A.; Gustavsson, T. Fluorescence Properties of DNA Nucleosides and Nucleotides: A Refined Steady-state and Femtosecond Investigation, J. Phys. Chem. B 2002, 106, 11367-11374.

(30) Markovitsi, D.; Sharonov, A.; Onidas, D.; Gustavsson, T. The Effect of Molecular Organisation in DNA Oligomers Studied by Femtosecond Fluorescence Spectroscopy, ChemPhysChem 2003, 3, 303-305.

(31) Markovitsi, D.; Gustavsson, T.; Banyasz, A. Absorption of UV Radiation by DNA: Spatial and Temporal Features, Mutat. Res. Rev. 2010, 704, 21-28.

(32) Middleton, C. T.; de La Harpe, K.; Su, C.; Law, Y. K.; Crespo-Hernandez, C. E.; Kohler, B. DNA Excited-State Dynamics: From Single Bases to the Double Helix, Ann. Rev. Phys. Chem. 2009, 60, 217-239. 
(33) Doorley, G. W.; McGovern, D. A.; George, M. W.; Towrie, M.; Parker, A. W.; Kelly, J. M.; Quinn, S. J. Picosecond Transient Infrared Study of the Ultrafast Deactivation Processes of Electronically Excited B-DNA and Z-DNA Forms of [poly(dG-dC)]2, Angew.

Chem. Int. Ed. 2009, 48, 123-127.

(34) Vayá, I.; Miannay, F.-A.; Gustavsson, T.; Markovitsi, D. High-Energy LongLived Excited States in DNA Double Strands, ChemPhysChem 2010, 11, 987-989.

(35) Sobolewski, A. L.; Domcke, W. On the Mechanism of Nonradiative Decay of DNA Bases: $a b$ initio and TDDFT Results for the Excited States of $9 \mathrm{H}$-Adenine, Eur. Phys. J. D: Atom., Mol. and Opt. Phys. 2002, 20, 369-374.

(36) Bensasson, R. V.; Land, E. J.; Truscott, T. G. Excited States and Free Radicals in Biology and Medicine; Oxford University Press: Oxford, 1993.

(37) Salet, C.; Bensasson, R. Studies on Thymine and Uracil Triplet Excited State in Acetonitrile and Water, Photochem. Photobiol. 1975, 22, 231-235.

(38) Hare, P. M.; Middleton, C. T.; Mertel, K. I.; Herbert, J. M.; Kohler, B. Timeresolved Infrared Spectroscopy of the Lowest Triplet State of Thymine and Thymidine, Chem. Phys. 2008, 347, 383-392. 


\section{Corresponding Author}

Thomas Gustavsson, email: thomas.gustavsson@ cea.fr

\section{Biographies}

Thomas Gustavsson received his $\mathrm{PhD}$ in Physics at the University of Stockholm (Sweden) in 1988. He joined CNRS in 1991 where he is presently research director. He developed and applied various techniques of ultrafast laser spectroscopy for studies of solution phase chemistry. He is currently focusing his activity on non-radiative processes in photoexcited DNA.

Roberto Improta received his $\mathrm{PhD}$ in Chemistry at the University Federico II in Naples (Italy) in 1998. After post-doctoral work with Prof. V. Barone, he joined the CNR (Italy) in 2001 where he now works as an independent researcher. He is presently visiting scientist at the Francis Perrin Laboratory. He is specialized in the development and in the application of quantum chemical methods to the study of large molecules in solution

Dimitra Markovitsi studied chemical engineering at the National Technical University of Athens (Greece). She received her PhD in Chemistry from the "Louis Pasteur" University (Strasbourg, France). She joined the CNRS in 1981 where she is currently research director and Head of the Francis Perrin Laboratory (http://www-lfp.cea.fr/). She has an expertise in photophysics and photochemistry of organized molecular systems. At present her research projects are focused on the interaction of UV radiation with DNA. 